\title{
ORIGINAL ARTICLE \\ The effect of low-frequency TENS in the treatment of neuropathic pain in patients with spinal cord injury
}

\author{
EC Celik ${ }^{1}$, B Erhan ${ }^{1}$, B Gunduz ${ }^{1}$ and E Lakse ${ }^{2}$
}

\begin{abstract}
Study design: Prospective, randomized and controlled study.
Objectives: The aim of the study was to investigate the effect of low-frequency transcutaneous electrical nerve stimulation (LF-TENS) in the treatment of neuropathic pain in patients with spinal cord injury (SCl).

Methods: A total of $33 \mathrm{SCl}$ patients with neuropathic pain were included in the study. History, duration, localization and characteristics of pain were recorded. Visual analog scale (VAS) was used to investigate the effect of LF-TENS four times during the day. Patients were randomly assigned to study and control groups. The study group was treated with $30 \mathrm{~min}$ of LF-TENS daily for 10 days while the placebo group with 30 min of sham TENS.
\end{abstract}

Results: The mean age of the patients was $36.55 \pm 10.36$ years. Out of 33 patients, 7 were tetraplegic and 26 were paraplegic. Twenty-three patients had complete SCI while 10 patients had incomplete injuries. Two groups were similar with respect to age, gender, duration, level and severity of injury. In the LF-TENS treatment group, a statistically significant reduction of the VAS values was observed, however, such an effect was not evident in the control group.

Conclusion: This study revealed that in treatment of neuropathic pain of SCI patients, LF-TENS may be effective.

Perspective: This article presents LF-TENS may effectively complement pharmacological treatment in patients with $\mathrm{SCl}$ and neuropathic pain.

Spinal Cord (2013) 51, 334-337; doi:10.1038/sc.2012.159; published online 8 January 2013

Keywords: neuropathic pain; spinal cord injury; low-frequency TENS

\section{INTRODUCTION}

Unfortunately, pain is a common complication following spinal cord injury (SCI) with prevalence of 18 to $96 \%$, and approximately $30 \%$ of these manifests as neuropathic pain. ${ }^{1}$ One-third of these patients rate their pain as severe. Persistent pain following SCI interferes with important daily activities including sleep, and makes it difficult for patients to achieve an acceptable quality of life following their injury. ${ }^{2}$

Neuropathic pain syndromes represent a group of highly heterogeneous clinical conditions. Such heterogeneity is apparent from the presentation of patients with various pain symptoms, including spontaneous pain, continuous pain, paroxysmal pain or evoked pain, which can be differentiated better with clinical examination. ${ }^{3,4}$ Leeds assessment of neuropathic symptoms and signs pain scale (LANSS) can be used for differentiation between neuropathic pain and nociceptive types of pain. The sensitivity and specificity of the Turkish version of LANSS was evaluated by Yucel $e t a l^{4}$

SCI-related neuropathic pain is often difficult to relieve. ${ }^{5-8}$ A wide variety of treatments have been analyzed to manage neuropathic pain. Various pharmacologic treatments such as antidepressants, anticonvulsants, alpha-adrenergic agonists, opioids, local anesthetics and $N$-methyl-D-aspartate receptor antagonists have been used in stepwise progression. ${ }^{5,8,9}$ As a result of the limited pain relief provided by pharmacological treatments or because of severity of the side effects experienced in patients with SCI and neuropathic pain with pharmacological treatments, non-pharmacological treatments such as physical therapy, relaxation, and acupuncture are also suggested., ${ }^{6,8,10-12}$
Transcutaneous electrical nerve stimulation (TENS) is an inexpensive, noninvasive, self-administered technique that is used as an adjunct to medication. ${ }^{13,14}$ Treatments with TENS are rarely associated with negative side effects and have been reported to be effective in patients with neuropathic pain. ${ }^{6,8,11,12}$ Most studies have assessed the effect of either of high- or low- frequency TENS (LF-TENS). ${ }^{6}$

Our aim is to investigate the effect of LF-TENS for the treatment of neuropathic pain in SCI patients in a case-control, single blind, prospective study.

\section{MATERIALS AND METHODS}

Patients older than 18 years took part; and patients suffering from pain other than neuropathic pain, urinary infections, heterotrophic ossification, pressure ulcer and severe spasticity were not included in the study. This study included 33 SCI inpatients with neuropathic pain at or below the level of cord injury.

Neuropathic pain was diagnosed by the examination of an experienced physiatrist and confirmed with a LANSS score of 12 and above.

All patients were examined and classified according to ASIA/IMSOP 2000 International Neurological Examination and Classification Standards. Patients' demographic characteristics, history, duration, localization and characteristics of pain were recorded.

All patients were prescribed to take $10 \mathrm{mg}$ per day of amitriptyline at 2000 hours for at least 15 days for a stable effect, before treatment. We refrained from using any other medication for pain. Patients were then randomly assigned as study and control groups (Figure 1) Patients signed a written consent on their first visit. Enrolled patients were assessed and assigned to

${ }^{1}$ Department of Physical Medicine and Rehabilitation, Istanbul Physical Medicine and Rehabilitation Training Hospital, Istanbul, Turkey and ${ }^{2}$ Bursa Medical Park Hospital, Bursa, Turkey Correspondence: Dr EC Celik, Istanbul Physical Medicine and Rehabilitation Training Hospital, Atakent mah 3 Etap D28 Blok Daire 9 Kucukcekmece Istanbul, Istanbul, Turkey, E-mail: evrimcoskun@yahoo.com

Received 9 January 2012; revised 22 October 2012; accepted 28 October 2012; published online 8 January 2013 


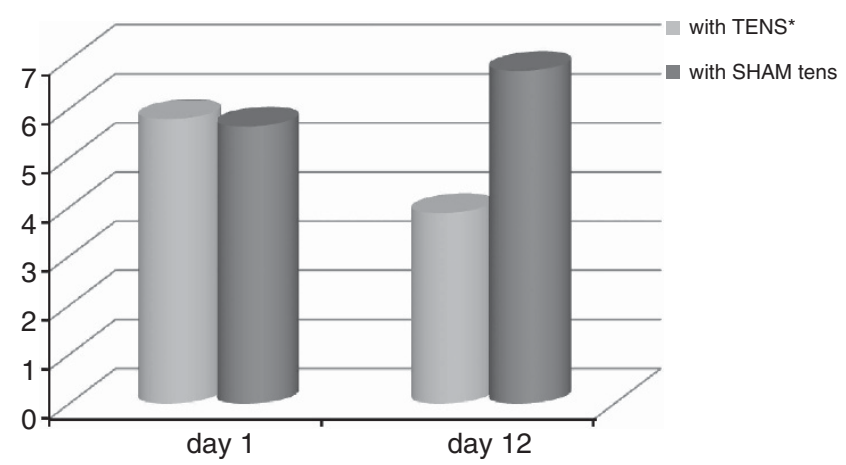

Figure 1 First- and twelfth-day mean VAS scores of TENS and sham TENS groups.

either LF-TENS or sham TENS stimulation according to the protocol (every other patient enrolled was assigned to start with LF-TENS).

All TENS applications were performed by the same physiatrist between 0800 and 1200 hours. Two electrodes were placed to the proximal and two to the distal parts of the region with neuropathic pain. We used two channels with four electrodes, which had $20 \mathrm{~mm}$ weight and $40 \mathrm{~mm}$ length. Electrodes were located separately. Patients in the study group were treated daily with LF-TENS (pulse frequency $4 \mathrm{~Hz}$, pulse duration $200 \mu$ s and pulse amplitude $50 \mathrm{~mA}$ ), whereas the placebo group were treated with sham TENS (electrodes placed, no stimulation ) for a duration of $30 \mathrm{~min}$ for 10 days. Both TENS appliances were turned on and counting down from $30 \mathrm{~min}$. All patients had neuropathic pain at and below SCI level, so patients could not tell if stimulus was being given or not. Patients had not been instructed on how to change stimulation programs within the machine and we ensured they did not attempt to do this.

We wanted the patient to measure their pain severity with visual analog scale (VAS) four times a day (morning, noon, evening and night) on the first and the twelfth day of the study. The physiatrist who helped patients measuring VAS scores, did not know whether patients were in the TENS or sham TENS group. At the beginning VAS, values were used as a baseline measurement of pain. After, TENS and sham TENS were used for treatment for 10 days, Finally, second set of VAS values were recorded.

In addition to descriptive statistics, the Wilcoxon and Spearman correlation tests were used to evaluate the results of the groups, and the Mann-Whitney $U$-test was used for comparison of the two groups. $P \leq 0.05$ was accepted as statistically significant.

We certify that all applicable institutional and governmental regulations concerning the ethical use of human volunteers were respected during the course of this research. Also informed consent was obtained from each subject.

\section{RESULTS}

Thirty-three patients (24 men and 9 women) participated in the study. Patients' demographic characteristics are summarized in Table 1. Mean age was $36.55 \pm 10.36$ years.

There was no statistical difference between groups in terms of age and gender.

Seven patients were tetraplegic and 26 patients were paraplegic (Table 2).

Average period after injury was 24.12 months (2-27 months) for the patients studied. Average time lapse between the injury and the development of pain was 5.02 months (0.03-48 months), and average pain duration was 19.10 months (1-170 months). Locations of neuropathic pain are seen in Table 3 . When locations and duration of pain, average time lapse between the injury and pain were compared, no statistically significant differences were observed between the groups.

Mean LANSS score was 16.94 (s.d. \pm 2.55 ) with range 13-23.
Table 1 Patient demographics

\begin{tabular}{lccc}
\hline & All patients & Patients with & Patients with sham \\
Patient demographics & $(\mathrm{n}$, 33) & TENS (n, 17) & TENS (n, 16) \\
\hline Age (mean \pm s.d.) & $36.55 \pm 10.36$ & $38.18 \pm 9.86$ & $34.81 \pm 10.91$ \\
Female & 9 & 4 & 5 \\
Male & 24 & 13 & 11 \\
\hline
\end{tabular}

Abbreviation: TENS, transcutaneous electrical nerve stimulation.

Table 2 Characteristics of spinal cord injury

\begin{tabular}{lccc}
\hline Level of injury & $\begin{array}{c}\text { All patients } \\
(\mathrm{n}, 33)\end{array}$ & $\begin{array}{c}\text { With TENS } \\
(\mathrm{n}, 17)\end{array}$ & $\begin{array}{c}\text { With sham } \\
(\mathrm{n}, 16)\end{array}$ \\
\hline TENS & 7 & 4 & 3 \\
Tetraplegia $(n, \%)$ & 26 & 13 & 13 \\
Paraplegia $(n, \%)$ & & & \\
Type of injury & & 13 & 10 \\
$\begin{array}{l}\text { Complete injury }(n, \%) \\
\text { Incomplete injury }(n, \%)\end{array}$ & 23 & 4 & 6 \\
\hline
\end{tabular}

Abbreviation: TENS, transcutaneous electrical nerve stimulation.

Table 3 Location of pain

\begin{tabular}{lccccc}
\hline & Foot & $\begin{array}{c}\text { Knee and } \\
\text { cauda }\end{array}$ & $\begin{array}{c}\text { Femur and } \\
\text { caudal }\end{array}$ & $\begin{array}{c}\text { Back and } \\
\text { caudal }\end{array}$ & $\begin{array}{c}\text { Cervical and } \\
\text { caudal }\end{array}$ \\
\hline $\begin{array}{l}\text { With TENS } \\
(n, 17)\end{array}$ & 2 & 1 & 5 & 7 & 2 \\
$\begin{array}{l}\text { With sham TENS } \\
(n, 16)\end{array}$ & 1 & 2 & 5 & 7 & 1 \\
\begin{tabular}{l} 
Total $(n, 33)$ \\
\hline
\end{tabular} & 3 & 3 & 10 & 14 & 3 \\
\hline
\end{tabular}

Abbreviation: TENS, transcutaneous electrical nerve stimulation.

On the first day, neuropathic pain mean VAS value was $5.79 \pm 2.17$ in the LF-TENS group and $5.64 \pm 1.81$ in the sham TENS group. No significant difference was observed when the first-day VAS mean values of the two groups were compared $(P=0.387)$. On the twelfth day (after 10 days of treatment), neuropathic pain mean VAS value was $3.88 \pm 2.5$ in the LF-TENS group and $6.77 \pm 1.42$ in the sham TENS group. After 10 days of treatment, a statistically significant difference was observed in mean VAS values $(P=0.032$; Figure 1$)$.

In the TENS group, morning mean VAS value was $5.76 \pm 2.95$ on the first day while $3.35 \pm 2.62$ on the twelfth day; noon mean VAS value was $5.65 \pm 2.50$ on the first day while $3.82 \pm 2.68$ on the twelfth day, evening mean VAS value was $5.88 \pm 1.93$ on the first day while $3.94 \pm 2.46$ on the twelfth day; night mean VAS value was $5.88 \pm 3.06$ on the first day while $4.41 \pm 2.76$ on the twelfth day. A statistically significant reduction was observed between first-day and twelfth-day values, especially for the morning $(P=0.001)$, noon $(P=0.015)$ and evening evaluations $(P=0.078)$. However, there was no statistically significant difference between first day's and twelfth day's night VAS values in TENS group ( $P=0.078$; Figure 2$)$.

No side effects of LF-TENS were seen.

\section{DISCUSSION}

Nearly half of SCI patients are at risk of developing neuropathic pain. ${ }^{6,8}$ SCI-related neuropathic pain is often difficult to relieve. ${ }^{6,8}$ 


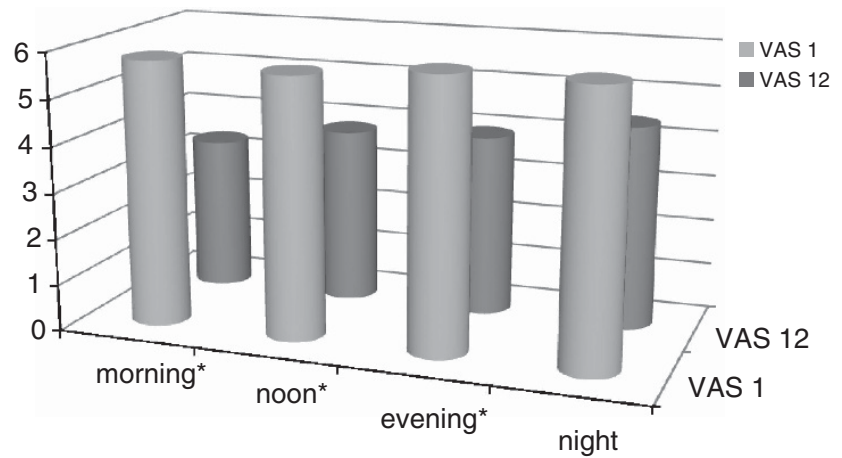

Figure 2 Comparison of first- and twelfth-day mean VAS scores of TENS group.

Treatment with TENS is rarely associated with negative side effects and has been reported to be effective in patients with neuropathic pain. ${ }^{6,8}$ Most studies have assessed the effect of either high-frequency TENS or LF-TENS. ${ }^{6,8}$ But few case-control studies were done about neuropathic pain and TENS. We researched the effects of LF-TENS for the treatment of neuropathic pain in SCI patients in a case-control, prospective study. The results of this study revealed that LF-TENS reduced neuropathic pain intensity in the morning, noon and evening but not at night, in SCI patients.

In the literature, $63.3 \%$ of patients with SCI pain had tried nonpharmacological treatment. TENS is one of the most tried nonpharmacological treatments. ${ }^{15}$ Norrbrink et al. made a large, cohort survey study on the use of non-pharmacological treatments (such as acupuncture, TENS, massage, heat, physical therapy and manipulation), for treating all types of pain in SCI patients, with questionnaires. The study evaluated how many patients used or were still using non-pharmacological treatments, and how efficient they were. Norrbrink et al. assessed that non-pharmacological treatments were tried out by a large number of patients with SCI pain. Massage and heat were rated most effective for relief of pain. Treatment with acupuncture and TENS had a success rate each of $28 \% .^{10,15}$

Fenollosa et al. ${ }^{16}$ devised a mixed 'step-by-step' approach, combining a pharmacological treatment and physical therapy by TENS. Thirty-three SCI patients with chronic pain were included in a four-phase study. The phase two (treatment with TENS) had the most significant improvement among the populations studied. ${ }^{10}$

Norrbrink et al. ${ }^{6}$ assessed the effects of high-frequency TENS and LF-TENS for neuropathic pain following SCI. Neither LF-TENS nor high-frequency TENS had a statistically significant effect on SCI patients with neuropathic pain, but they found a tendency for positive effect of TENS. The limitation of the study was the lack of a control group. In our study, we used sham TENS as the control group and LF-TENS for the treatment group. A statistically significant reduction was observed in VAS values when first- and twelfth-day values of the treatment group were compared.

Strömer et al. ${ }^{17}$ reported that $86 \%$ of pain and dysesthesia were localized below the lesion of SCI, $8 \%$ of pain was localized in the transition zone and only $6 \%$ of pain was localized in the nonparalyzed part of the body. Our study included only patients with neuropathic pain at or below the level of injury. Therefore, patients could not sense whether or not electrical stimulation was being given. Further studies should be designed to evaluate treatment of neuropathic pain of SCI in other locations (for example, above the level of SCI).
Demirel et al. ${ }^{18}$ demonstrated that chronic pain was more intense in the evening than in the morning and noon in SCI. In our previous study, we reported that neuropathic pain intensity was higher in the night than the morning, the noon and the evening. ${ }^{19}$ So to investigate the effect of LF- TENS during day time, we used VAS four times daily, for the evaluation of pain severity, before and after treatment. Administration of LF-TENS reduced pain intensity in the morning, the noon and the evening but not at the night, although patients were using their medication at night.

Somers and Clemente ${ }^{14}$ have also reported an animal study about the positive effects of TENS on neuropathic pain in rats.

When long-term effects of TENS were assessed in patients with chronic pain, positive results were found with respect to being able to resume work, home and social activities; increased activity level and pain management; and lower use of drugs and other treatments. ${ }^{6}$ Unfortunately, we have not measured these effects of TENS, and we did not assess the effect of LF-TENS beyond 15 days.

Constraints of this study are low participant number and inability to study long-term effect of LF-TENS. However, it is still important as a placebo-controlled study. Determination of the effect of given treatment to pain severity in the different intervals of the day is another important aspect of this study. After this study, evaluation of day-time pain severity is an important issue in pain treatment. Further studies associated with different frequencies of TENS are needed.

\section{CONCLUSIONS}

Until treatment options for SCI-related neuropathic pain become adequate, all interventions that might help a patient should be considered. LF-TENS may effectively complement pharmacological treatment in patients with SCI and neuropathic pain.

\section{DATA ARCHIVING}

There were no data to deposit.

\section{CONFLICT OF INTEREST}

The authors declare no conflict of interest.

\section{ACKNOWLEDGEMENTS}

This study was done with the inpatient in Istanbul Physical Medicine and Rehabilitation Training Hospital, Istanbul, Turkey.

1 Putzke JD, Richards JS, Hicken BL, Ness TJ, Kezar L, DeVivo M. Pain classification following spinal cord injury: the utility of verbal descriptors. Spinal Cord 2002; 40 118-127.

2 Widerström-Noga EG, Turk DC. Types and effectiveness of treatments used by people with chronic pain associated with spinal cord injuries: influences of pain and psychosocial characteristics. Spinal Cord 2003; 41: 600-609.

3 Calmels P, Mick G, Perrouin-Verbe B, Ventura MSOFMER (French Society for Physica Medicine and Rehabilitation). Neuropathic pain in spinal cord injury: identification, classification, evaluation. Ann Phys Rehab Med 2009; 52: 83-102.

4 Yucel A, Senocak M, Kocasoy Orhan E, Cimen A, Ertas M. Results of the Leeds Assesment of neuropathic Symptoms and Signs Pain Scale in Turkey: a validation study. J Pain 2004; 5: 427-432.

5 Ahn SH, Park HW, Lee BS, Moon HW, Jang SH, Sakong J et al. Gabapentin effect on neuropathic pain compared among patients with spinal cord injury and different durations of symptoms. Spine 2003; 28: 341-346.

6 Norrbrink C. Transcutaneous electrical nerve stimulation for treatment of spinal cord injury neuropathic pain. J Rehabil Res Dev 2009; 46: 85-94.

7 Perry KN, Nicholas MK, Middleton J. Spinal cord injury-related pain in rehabilitation: cross-sectional study of relationships with cognition, mood and physical function. Eur J Pain 2009; 13: 511-517.

8 Siddall PJ. Management of neuropathic pain following spinal cord injury: now and in the future. Spinal Cord 2009; 47: 352-359.

9 Tan E. Noropatik Agri ve Tedavisi. Turkish J Neurol 2004; 10: 390-400. 
10 Fattal C, Kong-A-Siou D, Gilbert C, Ventura M, Albert T. What is the efficacy of physical therapeutics for treating neuropathic pain in spinal cord injury patients? Ann Phys Rehab Med 2009; 52: 149-166.

11 Sun R, Kuo-Hsuan C. Neuropathic pain: mechanisms and treatments. Chang Gung Med J 2005; 28: 597-604.

12 To TP, Lim TC, Hill ST, Frauman AG, Cooper N, Kirsa SW et al. Gabapentin for neuropathic pain following spinal cord injury. Spinal Cord 2002; 40: 282-285.

13 Johnson MI, Bjordal JM. Transcutaneous electrical nerve stimulation for the management of painful conditions: focus on neuropathic pain. Expert Rev Neurother 2011; 11: 735-753.

14 Somers DL, Clemente FR. The relationship between dorsal horn neurotransmitter content and allodynia in neuropathic rats treated with high-frequency transcutaneous electric nerve stimulation. Arch Phys Med Rehabil 2003; 84: 1575-1583.
15 Norrbrink Budh C, Lundeberg T. Non-pharmacological pain-relieving therapies in individuals with spinal cord injury: a patient perspective. Complement Ther Med 2004; 12: 189-197.

16 Fenollosa P, Pallares J, Cervera J, Pelegrin F, Inigo V, Giner M et al. Chronic pain in spinal cord injuried: statistical approach and pharmacological treatment. Paraplegia 1993; 31: 722-729.

17 Störmer S, Gerner HJ, Grüninger W, Metzmacher K, Föllinger S, Wienke C et al. Chronic pain/dysaesthesiae in spinal cord injury patients: results of a multicentre study. Spinal Cord 1997; 35: 446-455.

18 Demirel G, Yilmaz H, Gencosmanoglu B, Kesiktaș N. Pain following spinal cord injury. Spinal Cord 1998; 36: 25-28.

19 Celik EC, Erhan B, Lakse E. The clinical characteristics of neuropathic pain in patients with spinal cord injury. Spinal Cord 2012; 50: 585-589. 